\title{
Introduction générale : une réflexion sur la circulation
}

\section{Christophe IMBERT ${ }^{l}$}

Voilà donc un ouvrage qui a réuni une équipe d'une trentaine de personnes qui, à des degrés divers, y ont apporté leur contribution. Il s'inscrit dans la lignée de recherches sur la mobilité qui croisent les apports de la démographie et de la géographie et est centré sur la notion de circulation autour de laquelle gravitent diverses expressions: navettes ou migrations pendulaires, mobilités ou migrations circulaires, migrations temporaires, circulation migratoire et territoires circulatoires. Si leurs définitions se recoupent parfois, leur usage varie selon les époques et les champs thématiques ou disciplinaires. Navettes et migrations pendulaires font référence à ce qu'est la circulation a minima, à savoir, un mouvement répétitif entre deux lieux. Néanmoins, lorsque les allers-retours sont réalisés dans une journée, y compris dans le cadre de la pendularité à longue distance, la circulation ne s'accompagne pas de multi-résidence, ce qui rend particuliers ces types de mouvements. A travers les territoires circulatoires (Tarrius, 1989), l'accent est mis sur les savoir-faire mobilisés dans la circulation internationale entre des lieux offrant des ressources complémentaires. Proche de la précédente dénomination, la circulation migratoire ne renvoie pas seulement à une circulation humaine, mais à toute forme d'échanges (financiers, matériels, idéels) engendrés dans un contexte de migration internationale entre un pays d'origine et un pays d'accueil (Doraï et alii, 1998). Cette notion rejoint celle de migration circulaire qu'aujourd'hui l'Union européenne entend promouvoir (EMN, 2010). Selon elle, la migration circulaire (circular migration) concerne deux catégories de populations : les élites migrantes qui travaillent en Europe et qui souhaitent développer une activité dans leur pays d'origine; les résidents de pays tiers, là encore généralement rattachés à des élites, qui souhaitent venir pour un travail saisonnier, pour se former (comme des doctorants), pour des activités professionnelles dans les domaines de la culture, des sports ou de la recherche (EMN, 2010). Une distinction nette est établie avec la migration temporaire (temporary migration) qui est définie comme une «migration pour un motif et/ou un but spécifiques avec une intention de retour dans le pays d'origine ou de départ vers une autre destination » (EMN, 2010 : 7). Il s'agit là d'une vision idéologique et pour le moins réductrice de la circulation, comme le soulignent A. Morice et B. Michalon dans un article sur les saisonniers du secteur agricole (2008: 16) : «associer dans un même paradigme, comme double nécessité, la « circulation » et le travail saisonnier des étrangers : voilà qui semble être une régression par rapport au temps où, en Italie, en France ou ailleurs, quand le journalier descendu de la montagne ne trouvait plus à se louer quelque part, il conservait néanmoins sa nationalité et le droit d'aller chercher du travail dans d'autres fermes ». La circulation a bien existé avant l'Union Européenne et celle-ci semble faire peu de cas de ceux qui circulent à l'intérieur de ses frontières; même si cette institution ne se prive pas de mettre en avant la «libre circulation » comme étendard de son humanisme. À certains égards, la circulation était moins contrôlée par les pouvoirs régaliens hier qu'aujourd'hui, en raison moins d'une volonté politique de le faire que d'une absence de moyens coercitifs.

Sur un registre plus académique, la distinction n'est pas aisée entre ce qui est désigné par migrations circulaires et par migrations temporaires. La recherche francophone sur la France emploie généralement le terme de migrations temporaires dont les migrations saisonnières sont une émanation (Châtelain, 1976) quand celle sur les Suds parlera plus volontiers de migrations circulaires (Dureau et Dupont, 1994). Pour la littérature anglophone sur la

\footnotetext{
${ }^{1}$ Merci à Yann Scioldo-Zürcher pour ses nombreux conseils de lecture que sa générosité a souvent transformés en cadeaux. Je lui adresse aussi mes remerciements, ainsi qu'à Marie-Antoinette Hily pour leur lecture amicale et experte de cette introduction.
} 
circulation, R. Chapman et R.M. Prothero (1983: 598) font le même constat d'un foisonnement de termes: "return migration, circular migration, wage-labor migration, seasonnal mobility, sojourner movements, transhumance, commuting ${ }^{2} »$. Nous avons fait le choix de la dénomination la plus générique : circulation. W. Zelinsky (1971) a explicitement défini ce mot «qui a été utilisé pour recouvrir une grande variété de déplacements habituellement de courte durée, répétitifs ou cycliques, ayant en commun l'absence de toute intention déclarée d'un changement de résidence permanent ou durable » (Dupont et Dureau, 1994 : 805-806). Il reste à montrer tout l'intérêt d'une étude globale de la circulation, indépendamment de ses formes particulières encastrées dans des métiers, des pratiques sociales ou des situations familiales. À cet effet, on remarquera d'abord que la circulation est ce qui réunit les personnes qui vivent entre plusieurs lieux, ce qui a été considéré dans les sociétés européennes médiévales et modernes comme une transgression et comme le mode de vie de groupes spécifiques : vagabonds et nomades, marchands et colporteurs, jongleurs et troupes de théâtre, étudiants et pèlerins, bergers et moissonneurs, journaliers et compagnons.

\section{La circulation : une question à historiciser}

L'histoire de la circulation en Europe n'est plus vraiment à faire ; du moins est-elle assez bien connue pour chacun des groupes qui viennent d'être cités. Sa quantification reste cependant difficile dès que l'on souhaite remonter dans le temps. W. Zelinsky (1971) fournit un cadre théorique au développement historique de la circulation, par l'introduction de la notion de transition mobilitaire, nommée ainsi en référence à la transition démographique. Selon ce schéma, qui se veut universel et concernerait tout mouvement de population :

1. dans les sociétés pré-modernes, la circulation est d'un niveau faible, sur des espaces très restreints sauf pour certains groupes précis (marchands, religieux, guerriers, bandits, etc.) ;

2. aux balbutiements de la révolution industrielle, elle augmenterait en raison d'alternances entre travaux agricoles et emplois salariés en ville ;

3. elle continuerait d'augmenter durant la révolution industrielle en se complexifiant et se diffusant à des couches de plus en plus larges de la société ;

4. elle se complexifierait encore plus pour atteindre des niveaux inégalés à l'époque contemporaine par le développement de la circulation internationale des élites et de celle qui s'effectue pour des motifs non professionnels (tourisme, loisirs).

R. Chapman et R.M. Prothero (1983) ont émis des doutes sur l'universalité du déroulement de la transition mobilitaire en mettant en avant des contre-exemples issus de sociétés coloniales. De façon plus fondamentale, si cette représentation historique de la circulation est subtilement articulée à l'évolution des autres formes de mobilités et se fonde sur des exemples puisés dans de nombreuses études empiriques, elle propose d'une part, un découpage du temps qui par construction insiste trop sur les discontinuités (la modernisation comme une rupture) et d'autre part, une flèche du temps irréversiblement orientée vers plus de complexification dont la démonstration reste à faire. L'histoire de la circulation en Europe plaiderait pour un schéma plutôt cyclique, même si un recueil systématique de données quantitatives fait défaut pour le montrer. Comme le remarque L. Lucassen (2001), on n'a pas attendu l'invention de la vapeur et du charbon pour être très mobiles ; et on doit à L.P. Moch (1992) le soin d'en avoir apporté la preuve à plus grande échelle spatiale (l'Europe de l'Ouest) et temporelle (depuis 1650). D'après nos recherches, pour la France, le $19^{\mathrm{e}}$ siècle est très bien couvert, grâce à la monumentale enquête historique d'A. Châtelain (1976) sur les migrations temporaires

\footnotetext{
${ }^{2}$ Migrations de retour, migration circulaire, migration pour un travail salarié, migrations saisonnières, séjours hors du lieu de résidence, transhumances, navettes domicile-travail.
} 
injustement tombées dans l'oubli. Sont quantifiées et cartographiées à l'échelle des départements français onze formes de migrations temporaires, dont sept héritées de l'Ancien Régime : 1) viticoles, 2) de moissons, 3) forestières, 4) pastorales, 5) de petite activité, artisanale, commerciale ou de service, allant jusqu'à la mendicité, 6) de travailleurs temporaires dans les villes, 7) de ruraux précaires exclus peu adaptés à la vie en ville; et quatre liées à l'industrialisation et à l'urbanisation : 8) pour les cultures industrielles, 9) pour les cultures délicates spéculatives, 10) du bâtiment et des grands travaux publics, 11) déclinaison de la forme 6 qui concerne des travailleurs du secteur industriel. Nous sommes bien loin d'une transformation radicale du panorama des circulations avec la naissance, progressive il est vrai, d'une société urbaine et industrielle. La période de l'Ancien régime est aussi prolifique en recherches sur la mobilité dont la plupart révèlent une grande diversité de circulations. Histoire culturelle (Croix, 1999) et démographie historique (Dupâquier, 2002) ont croisé le fer par revues interposées au tournant des années 2000 pour savoir si les populations rurales étaient enracinées dans leur paroisse ou déjà ouvertes sur le monde extérieur aux XVIe et XVII ${ }^{e}$ siècles. S'il y a un désaccord profond sur l'intensité des migrations et leurs conséquences sur la vie des villages, étant supposé que les migrations entrainent une rupture avec le milieu d'origine et donc un déracinement ${ }^{3}$, un consensus sur le niveau élevé de la circulation existe toutefois : «les migrations saisonnières et temporaires, bien loin de pouvoir être invoquées comme arguments contre le modèle sédentaire, contribuent à le conforter, en permettant à une bonne partie des paysans pauvres (en particulier les montagnards) de se procurer les compléments de ressources indispensables pour que leur famille puisse subsister au village » (Dupâquier, 2002 : 129). Quant à A. Croix (1999 : 122), il explique «que ces apparents errants ou vagabonds [...] restent, parfois au moins, attachés à leurs racines, et qu'ils y ramènent leur expérience même si l'on peut espérer qu'ils trouvent d'autres canaux que l'interrogatoire de justice pour en faire part », et de citer plus loin plusieurs travaux qui décrivent un tableau très similaire à celui qu'a dressé A. Châtelain dans les premières parties de son ouvrage.

La circulation est très largement, dans l'histoire européenne, une caractéristique des populations pauvres qui ont su développer des stratégies de mobilité pour échapper ou survivre (selon les cas) aux crises frumentaires. Ce sont les recherches sur la répression du vagabondage qui en donnent la description la plus précise, dont nous restituons ici les principaux résultats. Les premiers textes de loi visant la répression du vagabondage sont promulgués en Angleterre et en France dans les années qui suivent la Grande Peste (respectivement en 1349 et 1351), laquelle a décimé les communautés paysannes sédentarisées depuis le XIe siècle en Europe de l'Ouest et dont la raréfaction de la maind'œuvre afférente a eu pour conséquence une pression haussière sur les salaires qui ellemême a mis sur la route des petits paysans et artisans à la recherche de meilleurs revenus (Castel, 1995; Cubero, 1998; Geremek, 1976). Derrière les dénominations propices à alimenter les peurs des populations rurales et urbaines ${ }^{4}$, se cachent souvent des travailleurs ; la frontière est ténue entre circulation et vagabondage ${ }^{5}$, et les accidents de la vie qui mènent de l'une à l'autre sont nombreux : le journalier du XVIIe siècle qui commet un menu larcin et est marqué au fer (Viret, 2006), l'ouvrier de l'artisanat itinérant ${ }^{6}$ du XV $^{\text {e }}$ siècle (Geremeck,

\footnotetext{
${ }^{3}$ P.-A. Rosental (1999) a largement apporté une réfutation à cette dichotomie migration-rupture vs. mobilitémaintien pour le XIX ${ }^{\mathrm{e}}$ siècle.

${ }^{4}$ «caymans», «ribaulX», «ruffians», «truans» pour ne citer que quelques expressions du XIVe siècle.

${ }^{5}$ Cette frontière est celle de la désaffiliation sociale qui fait passer du statut de travailleur itinérant à celui de vagabond «sans aveu », c'est-à-dire sans communauté d'appartenance (Castel, 1995).

${ }^{6}$ « Nous avons souvent rencontré, au cours de nos réflexions précédentes, le problème de la connexion entre monde du travail et monde du crime : Classes Laborieuses et classes dangeureuses, tel est le titre d'une livre sur
} 
1976) et le hobo (Anderson, 1993) des années 1920 qui tombent dans la mendicité en sont des exemples. La criminalisation du vagabondage doit surtout être comprise comme une mise au pas de l'armée de réserve que constituaient les vagabonds pour les économies médiévales, modernes et pré-industrielles (Damon, 1996). Enfin, la circulation dite des élites ${ }^{7}$ qui a marqué les récits de voyage sous l'Ancien régime et plus particulièrement le XVIII ${ }^{\mathrm{e}}$ siècle (Roche, 2003) n'en était pas moins contrôlée pour ce qui concerne les mobilités estudiantines et les pèlerinages au Moyen-Âge : les jeunes clercs formaient une jeunesse turbulente et le peuple des pèlerins constituait une population diverse dont les marges, parfois peu recommandables, étaient soumises autant que possible à un contrôle policier sévère, en dépit de la protection des autorités ecclésiastiques dont elles bénéficiaient généralement ${ }^{8}$.

N'allons cependant pas croire que la circulation ne fût dans les temps passés qu'une réponse à une crise de la main-d'œuvre ou se résumât à une manifestation anomique d'un déséquilibre économique. Bien au contraire, sa contribution à l'avènement du capitalisme industriel a été de premier ordre. De la fin du Moyen-Âge au XIX ${ }^{\mathrm{e}}$ siècle, le colportage fut un des moteurs des économies européennes : avec les hommes circulaient objets et savoir-faire comme l'a décrit L. Fontaine (1993), par exemple pour les livres, le peigne ou la montre, assez fréquemment marchandises de contrebande. Ils n'hésitaient pas parfois à parcourir de longues distances, à l'instar des Ecossais en Pologne ou des «gavachos», ces Auvergnats qui ont failli payer l'outrecuidance d'avoir eu le quasi-monopole de la boulangerie dans le Royaume d'Espagne au $\mathrm{XVI}^{\mathrm{e}}$ siècle. Originaires des vallées alpines, auvergnates, écossaises ou pyrénéennes, de véritables dynasties familiales de colporteurs se sont formées en plusieurs générations jusqu'à obtenir pignon sur rue dans bien des villes européennes. Pour autant, une partie des bénéfices était réinvestie au pays avec souvent pour conséquence une remise en cause des hiérarchies villageoises anciennes. On est à bien des égards très proches de ce qui est décrit dans les travaux sur la circulation migratoire contemporaine (Doraï et alii, 1998). À une autre échelle, les colporteurs, les marchands des mers, qui circulaient dans réseaux ou des diasporas $^{9}$, ont été des protagonistes de la première mondialisation (Braudel, 1979); Grataloup, 2007).

Cette (trop) brève étude historique de la circulation nous porte à adopter une attitude critique face au schéma de la transition mobilitaire proposé par W. Zelinsky. D'une part, il n'est pas évident que la circulation ait continuellement augmenté depuis les époques pré-industrielles en Europe : on serait plus enclin à adopter un point de vue braudélien qui, sans nier le mouvement de fond pluriséculaire, souligne l'importance des fluctuations des cycles de courte

Paris au XIXe siècle. Mais cette distinction même est, en fait, le problème à étudier : les frontières de ces classes sont estompées, fluctuantes et leurs attaches très fortes. » (Geremek,1976 : 294).

${ }^{7}$ On peut l'assimiler pour le XVIIIe siècle aux voyages qui sont classés en 1772 par un dénommé Schlözer, professeur de philosophie à Göttingen, en deux grandes catégories : les voyages d'affaires (voyages religieux, commerciaux et éducatifs) et les voyages pour voyager (galant, de sociabilité mondaine et érudit) (Roche, 2003 : 570).

8 «Les étudiants parcouraient le Monde en recherchant la cléricature à Paris, les humanités à Orléans, le droit à Bologne, la médecine à Salerne, la magie à Tolède... «et les bonnes mœurs nulle part ». Presque tous les étudiants vivaient de la charité publique et étaient tous plus ou moins obligés de poursuivre cette existence leur vie durant car les débouchés auxquels ils pouvaient prétendre étaient très restreints [...] Quant aux autres, ils devenaient « clercs errants », gagnant leur vie comme musiciens, chanteurs ou poètes ambulants [...] » (Vexliard, 1997: 51). D. Roche (2003: 580), sans en minimiser le rôle social, remet à sa juste mesure l'ampleur quantitative du phénomène : « une autochtonéité universitaire existe très tôt, et le phénomène de la peregrinatio academica (circulation entre les universités) est un fait limité, alors que la mobilité locale est forte ».

9 Cf. le numéro des Annales, Histoire, Sciences Sociales (2003/3) consacré aux réseaux de marchands. F. Trivellato (2003), par exemple, insiste sur la pérennité des échanges extra-diasporiques effectués entre marchands juifs de Livourne, Italiens de Lisbonne et hindous de Goa. 
et surtout de moyenne durées. D'autre part, du point de vue de la variété de ses formes et de ses motifs, l'hypothèse d'une complexification de la circulation ne tient pas l'examen des faits. En revanche, ses caractéristiques spatio-temporelles se sont considérablement diversifiées depuis le siècle des Lumières, et ce, semble-t-il, pour chacune de ses formes : par exemple, l'ouvrier agricole qui cueille les fruits dans la basse vallée du Rhône et qui jadis franchissait le Massif Central ou descendait la vallée de la Durance, traverse aujourd'hui la Méditerranée ou l'Europe d'est en ouest.

\section{La circulation : un objet géographique}

La circulation est un vieil objet géographique apparu dès la naissance de la géographie comme discipline académique. À la suite des écrits de Vidal de la Blache (encart 5.2), des «géographies de la circulation» ont été réalisées, parmi lesquelles on trouve l'article «Introduction à la géographie de la circulation » de H. Cavaillès publié en 1940 dans les Annales de géographie, paru une année après celui de R. Dion dans les Annales de la Société Géologique du Nord ${ }^{10}$. De l'après-guerre aux années 1970, plusieurs manuels sont dédiés à cette question, comme ceux de R. Capot-Rey (1946), M. Perpillou (1958) et R. Clozier (1963). Toutefois, il ressort d'une lecture des comptes-rendus qui leur ont été consacrés une montée en puissance, sur fond de rapide modernisation de la France de l'après seconde guerre mondiale, de la question des transports (des personnes et des marchandises) et de leurs infrastructures ${ }^{11}$. On pourrait considérer ce glissement de la circulation vers les transports comme un rendez-vous manqué de la géographie avec ce phénomène pourtant central de la discipline $^{12}$ car la circulation est consubstantielle de la relation entre les lieux, et est aussi constitutive de la relation entre les individus et les espaces qu'ils habitent.

Il suffit de puiser dans les exemples historiques évoqués plus haut pour se convaincre de la fonction essentielle de la circulation dans la mise en relation de territoires parfois distants. On insiste de façon trop exclusive sur l'importance des migrations dans le peuplement d'espaces «vierges » ou la colonisation d'espaces habités : ces conquêtes ne se seraient pas réalisées sans le concours d'une circulation intense entre anciennes et nouvelles terres. Non seulement la circulation permet l'appui logistique du peuplement mais elle contribue souvent à l'aménagement des terres explorées et à l'installation de commerces et de services dans les lieux colonisés. N. Anderson (1993) a décrit de façon très détaillée comment les Hobos, rejoignant chaque printemps depuis Chicago les chantiers de construction des chemins de fer, avaient contribué à la Conquête de l'Ouest, et au moins à en assurer le succès final. La circulation est également indissociable de l'expérience de la migration internationale : il est désormais largement admis que celle-ci ne consiste nullement à couper les liens avec le pays d'origine. En situation de migration, les allers-retours des «migrants » avec leur famille restée «là-bas » sont très communs; cela vaut aussi lorsque le lieu d'immigration est un territoire neuf, les travaux de Françoise Dureau sur les travailleurs qui s'installent dans les villes pétrolières colombiennes (Dureau et Florez, 2000) en fournissant une illustration. L'urbanisation n'échappe pas non plus au rôle de la circulation : ce qui a été présenté comme exode rural est un long processus se terminant par une «migration définitive » mais où interviennent de nombreuses migrations de retour dont P.-A. Rosental (1999), s'inspirant des travaux de T. Hägerstand, a montré la contribution à l'urbanisation de la France. La circulation n'a pas seulement nourri la relation entre deux lieux éloignés, les nombreux

\footnotetext{
${ }^{10}$ Avec pour titre « Géographie de la circulation dans la partie centrale de la région du Nord ».

${ }^{11}$ Le dernier manuel cité est paru en 3 tomes, chacun dédié à des voies spécifiques : 1 - circulation terrestre (Clozier), 2 - maritime (Vigarié) et 3 - aérienne (Dacharry).

${ }^{12}$ H. Cavaillès (1940) n'a pas totalement perdu de vue les questions «qui circule ?» et «pour quoi ?»: il hiérarchise les fonctions de la circulation depuis la commerciale vers la militaire, l'agricole, du « citadin vaquant à ses affaires » (p. 180), du pèlerin et du nomade.
} 
centres urbains qui ont pendant très longtemps dû leur développement à leur hinterland sont là pour témoigner: l'Histoire du Grand Paris de la Renaissance à la Révolution (Muchembled, 2010) offre une multitude d'exemples d'ouvriers, de forains, de maraîchers ou de nourrices qui sillonnaient les routes qui confluaient vers Paris. Du côté des espaces ruraux, cette pratique a longtemps permis d'assurer la subsistance de communautés villageoises fragilisées par les crises économiques et les calamités agricoles, l'exemple le plus éclatant étant celui des colporteurs. L'utilité d'une étude géographique de la circulation tient donc, premièrement, à ce qu'elle permet d'entrer dans la boîte noire de l'interaction spatiale, c'est-à-dire de pénétrer à l'intérieur des flux, ce qui est une des préoccupations de ce livre. Elle tient aussi dans l'intérêt porté à la relation entre cette pratique et la transformation des espaces mis en relation: cet effet sur les quartiers d'une ville ou sur les dynamiques foncières des villages peut se voir en particulier à travers les investissements faits lors des retours avec l'argent gagné par le déplacement.

La géographie compte de nombreux travaux qui interrogent le phénomène de circulation comme impliquant une relation particulière à l'espace. Habiter plusieurs lieux à la fois est une possibilité qui a suscité la curiosité de nombreux géographes, les mots ne manquent pas à cet égard : habiter multi-local (Duchêne-Lacroix, 2011) ou habiter poly-topique (Stock, 2006), espaces de vie polycentriques (Robette et Lelièvre, 2005), ancrages multiples (Imbert, 2005b) - plus spécifiquement sur la multi-résidence - système résidentiel (Dureau, 2002) ${ }^{13}$, sont autant de tentatives conceptuelles pour penser le rattachement d'un individu à plusieurs lieux. Sans entrer dans le détail d'un concept ô combien complexe et discuté, on constate que les géographes qui ont investi «l'habiter» ont pris de la distance avec une vision heideggérienne qui fait l'éloge de la sédentarité, et même de l'enracinement exclusif dans un lieu. Une des difficultés majeures du concept réside dans l'association habiter/résider, comme l'explique M. Giroud (2007: 60) : «Cette confusion dissimule et distille implicitement une façon normative d'habiter: habiter nécessiterait une demeure, sous-entendu de préférence fixe, stable, unique et solide. Cette définition écarte ainsi tous les groupes qui ne respectent pas ces conditions, que ce soit par choix (dans le cas de groupes nomades ou circulants, ou d'individus pratiquant la pluri-résidence) ou par contrainte (par exemple certains individus sans-domicile fixe) ». Quel que soit le terme employé, plusieurs questions émergent de ces réflexions sur la dispersion des lieux de vie, souvent hors du seul cercle des géographes : quel est le contenu social de chaque lieu (relations sociales, activité professionnelle, etc.)? Comment se répartissent les activités entre ces lieux ? Ce mode de vie peut-il s'inscrire dans la durée?

\footnotetext{
Ainsi, la circulation doit être replacée dans un contexte social, professionnel et familial afin d'éviter l'écueil d'une essentialisation qui tendrait à associer trop strictement une pratique avec un mode de vie figé. Notre détour par l'Histoire a voulu le montrer, la circulation n'est pas la propriété de groupes qui ne cesseraient de reproduire de génération en génération leur mode de vie. A. Croix relève à juste titre l'exagération de J. Collins qui conclut trop rapidement au dualisme de la société française sous l'Ancien régime où des sédentaires auraient coexisté avec une population mobile, flottante. Si du point de vue d'un village, cette affirmation a quelque fondement ${ }^{14}$, du point de vue des individus, c'est moins évident : il est rare de circuler toute une vie et, inversement, la circulation est un phénomène

${ }^{13}$ Cette liste n'a évidemment pas prétention à l'exhaustivité.

${ }^{14}$ Comme le fait remarquer A. Croix (1999), si la consultation d'archives sur les taxes perçues montre une dichotomie entre une petite bourgeoise villageoise bien installée et une population pauvre «flottante », rien n'indique que des individus de la première n'aient pas circulé à certaines périodes, courtes on le concède, de leur existence. Ce qui crée une différenciation est plus la reconnaissance juridique d'une domiciliation qu'une immobilité improbable, au moins pour certains membres de ces familles.
} 
qui touche aussi des «sédentaires ». Une fois encore, l'exemple des Hobos le montre, les frontières de ce groupe ne sont pas très hermétiques et il est fréquent d'en sortir (Anderson, 1983). On pourrait évoquer dans le même ordre d'idée les processus de « sédentarisation » de populations nomades en diverses parties du globe. Il n'est pas inutile de le rappeler : il n'y a pas d'un côté les «circulants » dont la circulation serait une des propriétés sociales de leur mode de vie et de l'autre des « immobiles » qui ne partiraient de leur lieu de résidence qu'en de rares et ponctuelles occasions. Pour le dire autrement, des groupes spécialisés dans cette pratique n'en ont pas l'exclusivité. Et quand bien même leur identité se fonderait sur cette pratique (comme chez les Hobos, les routiers ou les Rroms), il n'est pas certain que chaque individu qui forme le groupe circule toute sa vie. Mieux encore, dans bien des cas la circulation ne peut se perpétuer que par la présence au sein d'un groupe familial ou affinitaire de personnes immobiles. Telle était la vocation première du colportage où la mobilité des uns permettait l'immobilité des autres et donc le maintien de la «maison » dans le village. Enfin, la pratique elle-même évolue, lieux et durées de séjours pouvant changer en fonction du contexte économique, politique et environnemental (Boyer, 2004 ; Bergeon, 2011).

\section{La notion de situation de mobilité : une approche biographique, contextualisée et intégrée de la circulation}

Ce bilan des travaux sur la circulation, aussi éclectiques soient-ils, éclaire les objectifs initiaux du projet : chercher à étudier les caractéristiques spatio-temporelles de la circulation, c'est approcher le phénomène sans le réduire à ce qui fonde l'identité de certains groupes, c'est aussi prendre acte de l'évolution pluriséculaire qu'il a connue avec l'augmentation des vitesses de déplacement et la diffusion de l'urbanisation. Il s'agit donc de décrire le phénomène avant de chercher à en extraire les facteurs sociodémographiques discriminants, à comprendre son rôle dans la vie d'un individu et sa contribution aux dynamiques urbaines. L'originalité de ce livre réside dans les approches mises en œuvre pour aborder ces questions. Un premier angle consiste à considérer la circulation comme une stratégie contrainte par un contexte économique et social et définie généralement au sein d'un groupe familial (Chapman et Prothero, 1983; Dureau, 2002). En effet la circulation des individus doit être réinscrite dans un double contexte, non seulement celui de la position historique et économique d'une ville comme Lisbonne dans les échanges européens et mondiaux, mais aussi celui des stratégies familiales. Le cadre familial ne doit, il est vrai, pas être négligé tant il est générateur de circulations: les enfants dont les parents sont divorcés circulent entre les domiciles parentaux (Bonvalet et Clément, 2005 ; Le Gall, 2005). De même, la relation entre choix familiaux et professionnels est à interroger dans la mesure où une situation de pendularité à longue distance ou d'absences répétées d'un membre du ménage s'accompagne de contraintes, qui présentent souvent une dimension genrée (Bertaux-Wiame et Tripier, 2006 ; Legrand et Ortar, 2011), différentes de celles engendrées par le déménagement de l'ensemble des membres du ménage, dans le cadre d'une expatriation, par exemple (Dubucs, 2009).

Il convient aussi de replacer la circulation dans le déroulement de la vie des individus. La position qu'entend défendre ce livre est celle d'une approche biographique. Quelle place occupe la circulation dans une vie ? À quel(s) moment(s) intervient-elle ? Pour quelle durée et en fonction de quels autres événements, familiaux ou professionnels ? Un des objectifs majeurs de cet ouvrage est de saisir dans quelle mesure la circulation se construit à travers une expérience progressivement acquise du déplacement.

De ces deux premières exigences découle une troisième qui implique de replacer la circulation au sein des autres pratiques de mobilités. Nombreux sont les travaux qui montrent qu'une migration peut être soit précédée soit suivie d'une pratique de circulation entre ancien et nouveau lieu de résidence : elle présente un caractère réversible, pour paraphraser un article 
de H. Domenach et M. Picouet (1987). Le fait de circuler est alors l'expression momentanée de la relation à un lieu qui peut évoluer vers l'absence de fréquentation ou vers l'installation d'une résidence dans ce lieu. Ainsi la circulation ne prend sens que dans un rapport que les individus tissent avec les lieux qu'ils fréquentent, d'où la nécessité d'étudier l'ancienneté de la fréquentation des lieux conjointement aux pratiques que les individus y déploient à un moment de leur existence. Pour résumer, il s'agit de se demander si l'on habite les lieux entre lesquels on circule.

Situer la circulation dans un contexte, dans une biographie et dans les autres pratiques de mobilités, telles sont les trois dimensions que ce livre souhaite articuler à travers l'introduction de la notion de situation de mobilité. Cette expression recouvre tout à la fois l'idée de processus continu (comme dans l'expression "on hérite d'une situation»), de configuration d'un espace de vie (présente dans l'expression « faire état d'une situation»), d'acquisition d'une position dans le jeu social (ne dit-on pas «avoir une bonne situation »?) et de choix de mobilités faits en fonction d'une situation, familiale, économique et sociale. Le terme situation rejoint ici l'outil conceptuel de compréhension des choix résidentiels que définit Y. Grafmeyer (2011 : 46) : « [...] la référence à la situation sociale joue un rôle central car c'est par son intermédiaire et à son niveau que s'articulent concrètement différents registres et différentes échelles d'analyse: les déterminants sociaux (valeurs, normes, expériences passées, habitudes...) qui sont dans une large mesure au principe de la constitution des goûts; les ressources et les contraintes objectives qui dessinent à un moment donné l'espace des possibles; et enfin les motifs ou les raisons que les individus sont susceptibles d'invoquer pour justifier leurs décisions ». La notion de situation de mobilité permet, ainsi définie, d'embrasser toute la complexité des processus auxquels participe la circulation.

Voilà donc un programme dont ce livre, nous en sommes convaincus, respecte les grandes lignes. Peut-être certaines dimensions n'ont-elles pu être explorées aussi loin que nous le souhaitions, comme le rôle que joue la circulation dans les dynamiques urbaines de Lisbonne. Il n'en reste pas moins que le lecteur y trouvera réflexions théoriques, méthodologiques et leur traduction empirique qui sont à considérer comme un retour d'expérience livré pour des recherches futures.

Ses trois parties reflètent notre volonté d'inscrire la recherche empirique effectuée à Lisbonne (parties 2 et 3 ) dans les avancées méthodologiques récentes - plus particulièrement celles menées à Migrinter - dans le champ des mobilités (partie 1). La première fait donc état des productions scientifiques sur les trajectoires résidentielles (chapitre 1) et les pratiques spatiales (chapitre 2) dont on a vu qu'elles étaient indissociables de la circulation. À ceci s'ajoute le fait que les travaux sur la circulation sont généralement disséminés dans les champs relativement segmentés de la mobilité résidentielle - des migrations, plus largement et de la mobilité quotidienne. Cette partie puise sa substance dans les nombreux projets de recherche auxquels les divers contributeurs de cet ouvrage participent et ont participé. Ce n'est pas son moindre intérêt que de montrer en quoi ces projets forment un tout cohérent et fondent une approche originale de la relation entre mobilités et dynamiques urbaines. Cette partie a enfin le mérite de donner à voir dans quels champs s'inscrit la recherche empirique effectuée à Lisbonne dans le cadre du projet MEREV.

La deuxième partie est précisément consacrée à la présentation du terrain à travers, d'une part, une explicitation des enjeux liés à la circulation dans la métropole lisboète (chapitre 3) et d'autre part, l'exposé de la méthodologie d'enquête (chapitre 4). On y trouvera des éléments de contexte qui constituent autant de clés d'analyse des observations faites sur le terrain. À cet 
égard, les quatre phases de la collecte se sont étalées entre février 2009 et décembre 2010, dans une période de crise dont nous méconnaissons encore l'ampleur, mais dont les effets se faisaient ressentir de façon croissante sur les économies européennes et avec une acuité particulière sur l'économie portugaise. Cette partie est aussi le temps d'une réflexion méthodologique sur la mise en œuvre, dans un protocole d'enquête, de l'observation de situations de mobilité à l'échelle de biographies - nous en verrons les limites aussi.

Puis vient le temps de la restitution des résultats, qui fait l'objet de la troisième partie et qui débute, dans l'ordre logique des choses, par la construction d'une typologie des caractéristiques spatio-temporelles de la circulation (chapitre 5). La diversité constatée est dans un second temps mise en relation avec les disparités sociales dont elle témoigne (chapitre 6), les trajectoires résidentielles et familiales qui la structurent (chapitre 7) et la variété des pratiques des lieux fréquentés qui l'accompagne (chapitre 8). Le chapitre 9, déjà publié dans la revue électronique Articulo ${ }^{15}$ se distingue en ce qu'il est une étude transversale des dimensions qui définissent une situation de mobilité autour de la notion de pénibilité.

\footnotetext{
${ }^{15}$ Que nous remercions pour nous avoir aimablement donné son accord pour reproduire ici l'article.
} 